\title{
ON THE COMPLEXITY OF CLASSES OF UNCOUNTABLE STRUCTURES: TREES ON $\aleph_{1}$
}

\author{
SY-DAVID FRIEDMAN AND DÁNIEL T. SOUKUP
}

\begin{abstract}
We analyse the complexity of the class of (special) Aronszajn, Suslin and Kurepa trees in the projective hierarchy of the higher Baire-space $\omega_{1} \omega_{1}$. First, we will show that none of these classes have the Baire property (unless they are empty). Moreover, under $(V=L)$, (a) the class of Aronszajn and Suslin trees is $\Pi_{1}^{1}$-complete, (b) the class of special Aronszajn trees is $\Sigma_{1}^{1}$-complete, and (c) the class of Kurepa trees is $\Pi_{2}^{1}$-complete. We achieve these results by finding nicely definable reductions that map subsets $X$ of $\omega_{1}$ to trees $T_{X}$ so that $T_{X}$ is in a given tree-class $\mathcal{T}$ if and only if $X$ is stationary/non-stationary (depending on the class $\mathcal{T}$ ). Finally, we present models of $\mathrm{CH}$ where these classes have lower projective complexity.
\end{abstract}

\section{INTRODUCTION}

We set out to investigate the complexity of certain well-studied classes of $\aleph_{1}$ trees on $\omega_{1}$. In particular, under various set-theoretic assumptions, we determine the Borel/projective complexity of the class of Aronszajn, Suslin and Kurepa trees as a subset of the higher Baire space $\omega_{1}{ }^{\omega_{1}}$. In several cases, we prove the existence of nicely definable reductions between these tree classes and the stationary relation on $\mathcal{P}\left(\omega_{1}\right)$. As the latter is $\Pi_{1}^{1}$-complete under $V=L$, we get the parallel completeness of the tree classes. In the case of Kurepa-trees, we use a different coding argument.

The general setting of our paper is the higher Baire spact 11 on $\omega_{1}{ }^{\omega_{1}}$ and $2^{\omega_{1}}$. Basic open sets correspond to countable partial functions which, in turn, give rise to a $\omega_{1}$ Borel structure on $\omega_{1}{ }^{\omega_{1}}, 2^{\omega_{1}}$ and so $\mathcal{P}\left(\omega_{1}\right)$ as well. This allows us to measure the complexity of subsets of $\omega_{1}^{\omega_{1}}$ or, equivalently, of families of natural combinatorial structures on $\omega_{1}$. In this paper, we will focus on models of CH i.e., $2^{\aleph_{0}}=\aleph_{1}$. This is a fairly natural assumption in this higher Baire setting which, in particular, ensures that $\omega_{1}{ }^{\omega_{1}}$ has a basis of size $\aleph_{1}$. This, of course, is analogous to the standard Baire space $\omega^{\omega}$ having a countable basis.

Our primary interest lies in the set of $\aleph_{1}$-trees: partial orders $T=\left(\omega_{1},<_{T}\right)$ on $\omega_{1}$ so that (1) the set of predecessors of each node is well-ordered 3 and (2) for any ordinal $\xi$, the set of nodes $T_{\xi}$ of height $\xi$ is countable and non-empty if $\xi<\omega_{1}$ and empty otherwise.

Following [16], we will use TO to denote the class of trees without uncountable branches; so we allow trees with uncountable levels here. A tree $T$ in TO is called Aronszajn if $T$ is also an $\aleph_{1}$-tree (i.e., all levels are countable). We call $T$ a Suslin

Date: June 4, 2019.

${ }^{1}$ The name generalized Baire space is also commonly used.

${ }^{2}$ This allows us to define a height function on $T$ and the levels $T_{\xi}$ of $T$ 
tree if it is an Aronszajn tree without uncountable antichains. On the other hand, an $\aleph_{1}$-tree $T$ is Kurepa if it has at least $\aleph_{2}$ uncountable branches. We will denote the classes of these trees with AT, ST and KT, respectively. An Aronszajn tree is special if it is the union of countably many antichains. The latter collection will be denoted by sAT. These are the main classes of trees we will be analyzing in detail. Let us refer the reader to the classical set theory textbooks [14, 11] and to [23] for a nice introduction to trees of height $\aleph_{1}$; the latter survey emphasizes the connection of trees to topology and linear orders.

TO: trees with no uncountable branch

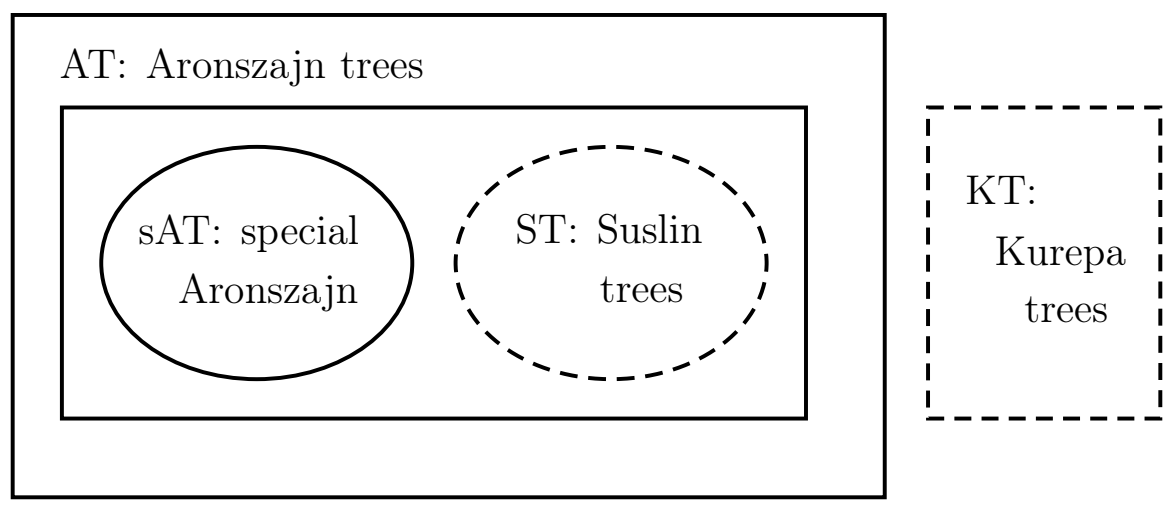

Figure 1. Classes of trees of height $\omega_{1}$

Recall that special Aronszajn trees exist in ZFC, however, even assuming the Continuum Hypothesis, ST and KT may be empty. In fact, as proved by R. Jensen, CH is consistent with AT $=\mathrm{sAT}[5]$ and so $\mathrm{ST}=\emptyset$ in this model 3 The consistency of no Kurepa trees was prove by Silver [21.

On the other hand, under $V=L$ (or just assuming strong enough diamonds), both Suslin and Kurepa-trees exist [1].

Let us present some results that will place our paper in the context of past research. Trees have played a significant role in the study of both the standard and higher Baire space [12, 16, 24, 6, 17]. Recall that the set of trees on $\omega$ without infinite branches is complete co-analytic. Analogously, a classical result from the theory of higher descriptive set theory is the following theorem of J. Väänänen.

Theorem 1.1. 16] CH implies that TO, the set of all trees on $\omega_{1}$ without uncountable branches, is $\Pi_{1}^{1}$-complete.

We mention that TO is $\Sigma_{1}^{1}$ if $M A_{\aleph_{1}}$ holds; indeed, $M A_{\aleph_{1}}$ implies that TO is exactly the set of special trees on $\omega_{1}$ [3] which is easily verified as a $\Sigma_{1}^{1}$ definition.

Yet another subclass of TO is the following: a canary tree $T$ is a tree of size continuum with no uncountable branches with the property that in any extension $W$ of the universe $V$ with $\mathbb{R}^{V}=\mathbb{R}^{W}$, if a stationary set of $V$ is no longer stationary in $W$

${ }^{3}$ We mention that AT $\neq$ sAT does not imply the existence of Suslin-trees [19]. 
then $T$ has an uncountable branch in $W$. Now, canary trees give a simple definition for a subset of $\omega_{1}$ to be stationary.

Theorem 1.2. 17, 10, There is a Canary-tree iff Stat $\subset \omega_{1}{ }^{\omega_{1}}$, the set of all stationary subsets of $\omega_{1}$, is $\Sigma_{1}^{1}$. Moreover, the existence of Canary trees is independent of GCH.

If $V=L$ then there are no canary trees and in fact, the following polar opposite result holds which appears implicitly in [7].

Theorem 1.3. [7] If $V=L$ then Stat is $\Pi_{1}^{1}$-complete.

We will use this theorem to show that certain classes of trees are complete in their complexity class. Finally, let us mention that there are strong connections between infinitary logic, trees and the complexity questions that our paper is concerned with [24, 20, 7]. We only included the results most relevant for our studies but we would like to refer the reader to the survey [24] and the book [8] for more details.

First, we will start by showing that non of the classes AT, sAT, ST and KT have the Baire property and hence they are non Borel (unless ST and KT are empty, in which case they are trivially Borel). Moreover, we will prove the following results about the complexity of these classes:

\begin{tabular}{|c|c|c|c|c|}
\hline & AT & sAT & ST & KT \\
\hline ZFC & $\Pi_{1}^{1} \backslash$ Borel & $\Sigma_{1}^{1} \backslash$ Borel & $\Pi_{1}^{1} \backslash$ Borel or $\emptyset$ & $\Pi_{2}^{1} \backslash$ Borel or $\emptyset$ \\
\hline$V=L$ & $\Pi_{1}^{1}$-complete & $\Sigma_{1}^{1}$-complete & $\Pi_{1}^{1}$-complete & $\Pi_{2}^{1}$-complete \\
\hline Abraham-Shelah model & $\Delta_{1}^{1} \backslash$ Borel & $\Delta_{1}^{1} \backslash$ Borel & $\Delta_{1}^{1} \backslash$ Borel & $?$ \\
\hline$M A_{\aleph_{1}}$ or $P F A(S)[S]$ & \multicolumn{2}{|c|}{$\Delta_{1}^{1} \backslash$ Borel } & $\emptyset$ & $?$ \\
\hline
\end{tabular}

Figure 2. A summary of complexity

Some of these results are easy consequences of known theorems (such as the results regarding the Abraham-Shelah model which we will describe shortly). However, the completeness of the classes under $V=L$ requires significant work and new ideas. We present the ZFC results and facts about the Abraham-Shelah model in Section 2, Then, in Section 3, we will show that stationarity can be reduced (in a Borel way) to the classes AT and ST. The results on $V=L$ will follow easily then. Finally, we deal with Kurepa-trees in Section 4. Note that under $M A_{\aleph_{1}}$ and $P F A(S)[S]$, AT $=$ sAT so the last line of Figure 2 follows by wrapping out the definitions 4 We end our paper with some remarks and open problems in Section 5 .

1.1. Preliminaries. We defined the tree classes already but let us review the most important descriptive set theoretic notions that we need. The family of Borel sets in $\omega_{1}{ }^{\omega_{1}}$ is the smallest family containing all open sets which is closed under taking complements and unions/intersections of size $\aleph_{1}$. It is easy to see that the set of $\aleph_{1}$-trees forms a Borel set with an appropriate coding of the order into a subset of $\omega_{1}$.

\footnotetext{
${ }^{4}$ In fact, in the latter model, all Aronszajn trees are club-isomorphic 25.
} 
Now, a subset $\mathcal{T}$ of $\omega_{1}^{\omega_{1}}$ is $\Pi_{1}^{1}$ (and called co-analytic) if there is an open $B \subset$ $\omega_{1}{ }^{\omega_{1}} \times \omega_{1}{ }^{\omega_{1}}$ so that $T \in \mathcal{T}$ if and only for all $g \in \omega_{1} \omega_{1},(T, g) \in B$. Complements of $\Pi_{1}^{1}$ sets, denoted by $\Sigma_{1}^{1}$, are called analytic sets. In Section 2, the reader can see elementary applications of this definition.

Finally, a subset $\mathcal{T}$ of $\omega_{1} \omega_{1}$ is complete for a complexity class $\Gamma$ iff $\mathcal{T} \in \Gamma$ and for any $\mathcal{S} \in \Gamma$, there is a continuous $\pi: \omega_{1} \omega_{1} \rightarrow \omega_{1}{ }^{\omega_{1}}$ so that $T \in \mathcal{S}$ if and only if $\pi(T) \in \mathcal{T}$. That is, no matter how we pick $\mathcal{S}$ in $\Gamma$, we can completely decide $\mathcal{S}$ by our single fixed set $\mathcal{T}$ and using an appropriate continuous map. In Section 3, we shall see this definition at work.

Let us also recall some classical guessing principles: $\nabla^{+}$asserts the existence of a sequence $\underline{A}=\left\{\mathcal{A}_{\alpha}: \alpha<\omega_{1}\right\}$ of countable sets so that for any $X \subset \omega_{1}$, there is a club $C \subset \omega_{1}$ so that $C \cap \alpha, X \cap \alpha \in \mathcal{A}_{\alpha}$ for any $\alpha \in C$. In this situation, we say that $\underline{A}$ witnesses $\diamond^{+}$.

We say that $\underline{N}=\left(N_{\alpha}\right)_{\alpha<\omega_{1}}$ is a $\diamond^{+}$-oracle over $P$ if

(1) $\underline{N}$ is an increasing sequence of countable elementary submodels of $H\left(\aleph_{2}\right)$,

(2) $\bar{P},\left(N_{\alpha}\right)_{\alpha<\beta} \in N_{\beta}$ for all $\beta<\omega_{1}$, and

(3) $\underline{N}$ witnesses $\diamond^{+}$.

Clearly, if $\diamond^{+}$holds then for any $P \in H\left(\aleph_{2}\right)$, there is a $\diamond^{+}$-oracle over $P$. Also, recall that $\diamond^{+}$implies that ST and KT are non-empty [11].

For later reference, we state a few consistency results, the first being a now classical theorem of R. Jensen.

Theorem 1.4. 5] Consistently, $C H$ holds and all Aronszajn-trees are special.

Jensen's argument was built on an elaborate ccc forcing (in fact, a completely new iteration technique). A more mainstream proof of this theorem is due to S. Shelah [19] using countable support iteration of proper posets.

Given two trees $S, T$, a club-embedding of $T$ into $S$ is an order preserving injection $f$ defined on $T \uparrow C=\bigcup\left\{T_{\alpha}: \alpha \in C\right\}$, where $C \subset \omega_{1}$ is a club (closed and unbounded subset), with range in $S$. A derived tree of $S$ is a level product of the form $\Pi_{i<n} S \cap s_{i}^{\uparrow}$ where the $s_{i}$ are distinct nodes from the same level of $S$. A fully Suslin tree is a Suslin tree with the property that all its derived trees are Suslin as well.

We will refer to the model in the next theorem as the Abraham-Shelah model.

Theorem 1.5. 1] Consistently, $C H$ holds and there is a fully Suslin tree $R$ and special Aronszajn tree $U$ so that, for any Aronszajn tree $T$, either

(1) $T$ club-embeds into $U$ or

(2) there is a derived tree of $R$ that club-embeds into $T$.

Moreover, there are only $\aleph_{1}$-many Suslin-trees modulo club-isomorphism 6

In essence, the above theorem says that any Aronszajn tree is either special or embeds a Suslin tree closely associated to $R$.

Finally, the fact that there might be no Kurepa trees was proved by J. Silver in 1971.

\footnotetext{
${ }^{5}$ Here, $S \cap s_{i}^{\uparrow}=\left\{t \in S: t \geq s_{i}\right\}$.

${ }^{6}$ It is an intriguing open problem if one can find a model with a single Suslin-tree (modulo club-isomorphism).
} 
Theorem 1.6. 21] If a strongly inaccessible cardinal is Lévy collapsed to $\omega_{2}$ then in the resulting model, there are no Kurepa trees.

1.2. Acknowledgments. The authors would like to thank the Austrian Science Fund (FWF) for the generous support through Grant I1921. The second author was also supported by NKFIH OTKA-113047.

\section{Aronszajn and Suslin trees}

To avoid some technicalities, let us restrict our attention to certain regular trees only from now on: those trees $T$ which are rooted, every node in $T$ has at least two immediate successors and $T$ is pruned i.e., for any $s \in T$ of height $\alpha$ and any $\beta<\omega_{1}$ above $\alpha$, there is some $t \in T$ of height $\beta$ that extends $s$. These are simple Borel conditions and we assume that our classes AT, ST and later KT consist of only regular trees.

Now, let us start the complexity analysis of these classes. Our first observation follows from the definitions immediately.

Observation 2.1. $\quad$ (1) The set of all $\aleph_{1}$-trees on $\omega_{1}$ is Borel.

(2) AT and ST are both $\Pi_{1}^{1}$ sets.

(3) $\operatorname{sAT}$ is $\Sigma_{1}^{1}$.

Proof. The proof is a fairly standard exercise in descriptive set theory. To demonstrate the definitions, we prove that $\mathrm{AT}$ is $\Pi_{1}^{1}$ and leave the rest to the interested reader. We need to find an open $B \subset \omega_{1}{ }^{\omega_{1}} \times \omega_{1}{ }^{\omega_{1}}$ so that $T \in$ AT if and only for all $g \in \omega_{1}{ }^{\omega_{1}}$, $(T, g) \in B$. Indeed, let $B$ denote the set of pairs $(T, g)$ so that $T$ is an $\aleph_{1}$-tree on $\omega_{1}$ and $g \in \omega_{1}{ }^{\omega_{1}}$ does not code an uncountable branch in $T$. Now, $B$ is open in the product of codes for $\aleph_{1}$-trees (a Borel set) and $\omega_{1}{ }^{\omega_{1}}$. Indeed, if $g$ does not code an uncountable branch then either $g$ codes a countable branch in $T$ (i.e., there is a level of $T$ without any element of $g$ ) or $g$ codes two incomparable elements. Both cases can be witnessed by fixing a countable initial segment of $T$ and $g$ and hence, $B$ is open. Now, an $\aleph_{1}$-tree $T$ is Aronszajn if and only if for any $g,(T, g) \in B$.

So in models where AT = sAT e.g., in Jensen's model of CH from Theorem 1.4 we get the following.

Corollary 2.2. Consistently, $C H$ holds and $\mathrm{AT}=\mathrm{sAT} \in \Delta_{1}^{1}$ and so $\mathrm{ST}=\emptyset 7$

Our next goal is to show that none of the classes AT, sAT and ST are Borel, that is, unless ST $=\emptyset$ in which case it is trivially Borel. We will apply the following well-known fact.

Lemma 2.3. Suppose that $T, T^{\prime}$ are countable, rooted, binary branching, and pruned trees of height $\beta<\omega_{1}$. Then $T$ and $T^{\prime}$ are isomorphic. In fact, any isomorphism $f: T_{\leq \alpha} \rightarrow T_{\leq \alpha}^{\prime}$ with $\alpha<\beta$ extends to an isomorphism $\bar{f}: T \rightarrow T^{\prime}$.

The proof is an easy back-and-forth argument that we omit. This allows us to prove a new, relatively straightforward result.

Lemma 2.4. Suppose that $T$ is a regular $\aleph_{1}$-tree and $\mathcal{U}$ is somewhere co-meager in the set of all regular trees on $\omega_{1}$. Then

\footnotetext{
${ }^{7}$ We remark here that $M A_{\aleph_{1}}$ implies AT $=$ sAT so they are both $\Delta_{1}^{1}$ however $\mathrm{CH}$ fails.
} 
(1) there is an isomorphic copy $S$ of $T$ in $\mathcal{U}$, and

(2) $\mathcal{U}$ contains a tree with an uncountable branch.

Proof. (1) Suppose that $\mathcal{U}=\left[S^{0}\right] \backslash \bigcup\left\{Y_{\xi}: \xi<\omega_{1}\right\}$ where each $Y_{\xi}$ is a nowhere dense set of trees. I.e., any countable tree $S$ has a countable end-extension $S^{\prime}$ so that any extension of $S^{\prime}$ into a tree on $\omega_{1}$ is not in $Y_{\alpha}$. Here, $\left[S^{0}\right]$ denotes the (basic open) set of all trees extending $S^{0}$.

Now, we construct an increasing sequence of countable trees $\left(S^{\xi}\right)_{\xi<\omega_{1}}$ and isomorphisms $f_{\xi}: S^{\xi} \rightarrow T_{<\delta_{\xi}}$. Given $\left(S^{\xi}\right)_{\xi<\zeta}$, we look at $S^{<\zeta}=\bigcup_{\xi<\zeta} S^{\xi}$. The latter is isomorphic to $T_{<\delta}$ where $\delta=\sup _{\xi<\zeta} \delta_{\xi}$ witnessed by

$$
f_{<\zeta}=\bigcup_{\xi<\zeta} f_{\xi}: S^{<\zeta} \rightarrow T_{<\delta} .
$$

Define an end extension $\overline{S<\zeta}$ of $S^{<\zeta}$ of height $\delta+1$ by adding upper bounds to exactly those branches $b \subset S^{<\zeta}$ so that the chain $f_{<\zeta}[b]$ has an upper bound in $T_{\delta}$. Clearly, there is an isomorphism $\overline{f_{<\zeta}}: \overline{S^{<\zeta}} \rightarrow T_{\delta+1}$ that extends $f_{<\zeta}$. Now, let $S^{\zeta}$ be an end-extension of $\overline{S<\zeta}$ which cannot be extended to a tree on $\omega_{1}$ that is in $Y_{\zeta}$. This can be done since $Y_{\zeta}$ is nowhere dense. Finally, apply Lemma 2.3 to extend $\overline{f_{<\zeta}}$ to some isomorphism $f_{\zeta}: S^{\zeta} \rightarrow T_{<\delta_{\zeta}}$.

This finishes the construction and the tree $S=\bigcup\left\{S^{\zeta}: \zeta<\omega_{1}\right\}$ is as desired.

(2) Since there is a regular $\aleph_{1}$-tree $T$ which contains an uncountable branch, we can apply (1).

We shall use the fact that any non-meager set with the Baire property is somewhere co-meager. The previous lemma and latter fact immediately yields the following corollaries.

Corollary 2.5. (1) The isomorphism class of any regular $\aleph_{1}$-tree $T$ is everywhere non-meager.

(2) Suppose that T, S are non-isomorphic $\aleph_{1}$-trees. Then their isomorphism classes cannot be separated by sets with the Baire-property.

(3) The set of trees isomorphic to a fixed tree without an uncountable branch is $\Sigma_{1}^{1}$ but does not have the Baire property and hence is not Borel.

(4) The sets AT and sAT do not have the Baire property. In turn, AT and SAT are not Borel.

(5) If $\mathrm{ST} \neq \emptyset$ then $\mathrm{ST}$ does not have the Baire property and so ST is not Borel.

(6) If $\mathrm{KT} \neq \emptyset$ then $\mathrm{KT}$ does not have the Baire property and so $\mathrm{KT}$ is not Borel.

Proof. (11) and (2) immediate from Lemma 2.4 (1).

(3) If such an isomorphism class has the Baire property then there is a somewhere co-meager set of trees all isomorphic to a fixed tree with no uncountable branch. This is not possible by Lemma $2.4(2)$.

(44), (5) and (6) again follow from Lemma 2.4 (2): these classes are closed under isomorphism classes so must be everywhere non-meager. If they are Baire then they are somewhere comeager and hence contain a tree with an uncountable branch and also a special Aronszajn tree. This leads to a contradiction in case of any of these classes. 
So, whenever there is a Suslin tree then the set of all Suslin-trees is not Borel (but always $\Pi_{1}^{1}$ ). Could it be analytic too? We show that this is independent (even assuming $\mathrm{CH})$.

Proposition 2.6. In the Abraham-Shelah model, $\mathrm{AT} \neq \mathrm{sAT} \in \Delta_{1}^{1}$ and $\mathrm{ST} \in \Delta_{1}^{1}$ as well.

Proof. Indeed, there are non-special Aronszajn trees (even Suslin-trees) and a tree $T$ is special if and only if it club-embeds no derived subtree of a fixed Suslin tree $S$. Since there are only $\aleph_{1}$-many such derived subtrees, this gives $\mathrm{sAT} \in \Pi_{1}^{1}$ and so $\operatorname{sAT} \in \Delta_{1}^{1}$ (using Observation 2.1) .

In the Abraham-Shelah model, there are only $\aleph_{1}$ many Suslin trees modulo club isomorphism so fix a representative of each class and collect them as $\mathcal{S}$. Now, being Suslin is characterized by being club-isomorphic to some element of $\mathcal{S}$ which in turn implies $\mathrm{ST} \in \Sigma_{1}^{1}$ and $\mathrm{ST} \in \Delta_{1}^{1}$ as well (using again Observation [2.1).

In the next section, we show that both $\mathrm{AT}$ and $\mathrm{ST}$ are $\Pi_{1}^{1}$-complete if we assume $V=L$.

\section{Reductions BEtWEen subsets of $\omega_{1}$ AND $\aleph_{1}$-TREeS}

Our first theorem in this section establishes a continuous reduction between stationarity and ST in a strong form.

Theorem 3.1. Suppose $\diamond^{+}$. There is a map $X \mapsto T^{X}$ from subsets of $\omega_{1}$ to the set of downward closed $\aleph_{1}$-subtrees of $2^{<\omega_{1}}$ so that

(1) if $X \cap \alpha=Y \cap \alpha$ then $T^{X} \uparrow \alpha=T^{Y} \uparrow \alpha$,

(2) if $X$ is stationary then $T^{X}$ is Suslin, and

(3) if $X$ is non-stationary then $T^{X}$ has an uncountable branch.

Note that by $\mathrm{CH}, 2^{<\omega_{1}}$ has size $\aleph_{1}$ so we can easily transform our trees to live on $\omega_{1}$. So, we immediately get the following corollary by Theorem 1.3 .

Corollary 3.2. If $V=L$ then AT and ST are both $\Pi_{1}^{1}$-complete.

Let us proceed with the proof of the theorem.

Proof of Theorem 3.1. Let is start by fixing a $\diamond^{+}$-oracle $\bar{N}$ i.e., a sequence of elementary submodels $\left(N_{\alpha}\right)_{\alpha<\omega_{1}}$ of $\left(H\left(\aleph_{2}\right), \in, \prec\right)$ so that $\left(N_{\alpha}\right)_{\alpha<\beta} \in N_{\beta}$ and $\bar{N}$ witnesses $\diamond^{+}$.

Given $X \subset \omega_{1}$, we construct the downward closed subtree $T^{X} \subset 2^{<\omega_{1}}$ level by level in an induction, so that $\left(T_{\alpha}^{X}\right)_{\alpha<\beta} \in N_{\beta+1}$ for all $\beta<\omega_{1}$. In each step, we shall add a new countable level to the tree constructed so far. In successor steps $\beta=\alpha+1<\omega_{1}$, we simply take the binary extension $T_{\beta}^{X}=\left\{s^{\frown} i: s \in T_{\alpha}^{X}, i<2\right\}$ of $T_{\alpha}^{X}$.

Now, assume $\beta \in \omega_{1}$ is a limit ordinal. If $\beta \in \omega_{1} \backslash X$ then we let

$$
T_{\beta}^{X}=\left\{b \in 2^{\beta} \cap N_{\beta}: b \uparrow \alpha \in T_{\alpha}^{X} \text { for all } \alpha<\beta\right\} .
$$

In other words, we continue all branches through $T_{<\beta}^{X}$ which are in $N_{\beta}$. Since $N_{\beta}$ is countable, this is a valid extension and is defined in $N_{\beta+1}$. Moreover, any $s \in T_{<\beta}^{X}$ has an extension in $T_{\beta}^{X}$ (i.e., the tree remains pruned).

Second, if $\beta \in X$ then, working in $N_{\beta+1}$, we make sure that 
(1) any $s \in T_{<\beta}^{X}$ has an extension in $T_{\beta}^{X}$, and

(2) for any $A \in N_{\beta}$ so that $A \subset T_{<\beta}^{X}$ is a maximal antichain, any new element $t \in T_{\beta}^{X}$ is above a node in $A$.

Since $N_{\beta}$ is countable and $N_{\beta}, T_{<\beta}^{X} \in N_{\beta+1}$, the level $T_{\beta}^{X}$ can be constructed in $N_{\beta+1}$ (just as in the classical construction of Suslin trees [14]).

This induction certainly defines an $\aleph_{1}$-tree $T^{X}$ for any $X \subset \omega_{1}$. The next two claims will conclude the proof of the theorem.

Claim 3.3. If $X$ is stationary then $T^{X}$ is Suslin.

Proof. Suppose that $A \subset T^{X}$ is a maximal antichain. Since $\bar{N}$ guesses $A$ at club many points, we can find some $\beta \in X$ so that $A \cap T_{<\beta}^{X} \in \mathcal{N}_{\beta}$ and $A \cap T_{<\beta}^{X}$ is a maximal antichain in $T_{<\beta}^{X}$. So, at stage $\beta$, we made sure that any $t \in T_{\beta}^{X}$ is above some element of $A \cap T_{<\beta}^{X}$. In turn, we must have $A \subset T_{<\beta}^{X}$ and so $A$ is countable.

Claim 3.4. If $X$ is non stationary then $T^{X}$ has an uncountable branch.

Proof. There is some club $B$ that is disjoint from $X$, and there is a club $C \subset \omega_{1}$ so that $C \cap \alpha, B \cap \alpha \in N_{\alpha}$ whenever $\alpha \in C$. In particular, $C \cap B \cap \alpha \in N_{\alpha}$ for any $\alpha \in C \cap B$. Let $\left\{\beta_{\alpha}: \alpha<\omega_{1}\right\}$ be the increasing enumeration of $C \cap B$.

We construct $t_{\alpha} \in T_{\beta_{\alpha}}^{X}$ so that

(1) $t_{\alpha^{\prime}}<t_{\alpha}$ for all $\alpha^{\prime}<\alpha<\omega_{1}$,

(2) $\left(t_{\alpha^{\prime}}\right)_{\alpha^{\prime}<\alpha} \in N_{\beta_{\alpha}}$, and

(3) $t_{\alpha}$ is the $\prec$-minimal element of $2^{\beta_{\alpha}}$ that extends all elements in the chain $\left(t_{\alpha^{\prime}}\right)_{\alpha^{\prime}<\alpha}$.

In limit steps, note that $\bar{N} \uparrow B \cap C \cap \beta_{\alpha}=\left(N_{\beta_{\alpha^{\prime}}}\right)_{\alpha^{\prime}<\alpha} \in N_{\beta_{\alpha}}$. So the sequence $\left(t_{\alpha^{\prime}}\right)_{\alpha^{\prime}<\alpha}$ is in $N_{\beta_{\alpha}}$ too since it can be uniquely defined from $\bar{N} \uparrow B \cap C \cap \beta_{\alpha}$. As $\beta_{\alpha} \notin X$, we sealed all branches that are in $N_{\beta_{\alpha}}$, so $t_{\alpha} \in T_{\beta_{\alpha}}^{X}$ as well. In turn, $\left(t_{\alpha^{\prime}}\right)_{\alpha^{\prime} \leq \alpha} \in N_{\beta_{\alpha}+1}$.

This proves the theorem.

It would be interesting to see whether a single Suslin-tree suffices to construct such a reduction or if weaker reductions (say between stationarity and AT) exist under weaker assumptions than $\diamond^{+}$.

Next, we present a variation that reduces non-stationarity to sAT.

Theorem 3.5. Suppose $\diamond^{+}$. There is a map $X \mapsto T^{X}$ from subsets of $\omega_{1}$ to the set of downward closed $\aleph_{1}$-subtrees of $2^{<\omega_{1}}$ so that

(1) if $X \cap \alpha=Y \cap \alpha$ then $T^{X} \uparrow \alpha=T^{Y} \uparrow \alpha$,

(2) if $X$ is stationary then $T^{X}$ is Suslin, and

(3) if $X$ is non-stationary then $T^{X}$ is a special Aronszajn tree.

Proof. The idea is very similar: we build $T^{X}$ level by level and aim for a Suslin tree at stages $\beta \in X$. However, if $\beta \in \omega_{1} \backslash X$ then we shall try to make $T^{X}$ special. In fact, we will add the new level $T_{\beta}^{X}$ so that any nice enough monotone map $\varphi: T_{<\beta}^{X} \rightarrow \mathbb{Q}$ that is also in $N_{\beta}$ has an extension to $T_{\beta}^{X}$. We will need to make sure that any new 
node at level $\beta$ works simultaneously for all such specializing maps which inspires the definition of a specializing pair below. Intuitively, we not just assign a rational number $\varphi(s)$ to a tree node $s$ but also a promise (in the form of a positive number $\delta(s))$ to keep all values $\varphi(t)$ close to $\varphi(s)$ whenever $t$ is above $s$. The details follow below.

Fix a $\diamond^{+}$-oracle $\bar{N}$. Given $X$, we construct the downward closed subtree $T^{X} \subset 2^{<\omega_{1}}$ so that $\left(T_{\alpha}^{X}\right)_{\alpha<\beta} \in N_{\beta+1}$ for all $\beta<\omega_{1}$. If $\beta$ is successor or if $\beta \in X$ then, working in $N_{\beta+1}$, we repeat the construction in the previour theorem. We make sure that

(1) any $s \in T_{<\beta}^{X}$ has an extension in $T_{\beta}^{X}$, and

(2) for any $A \in N_{\beta}$ so that $A \subset T_{<\beta}^{X}$ is a maximal antichain, any new element $t \in T_{\beta}^{X}$ is above a node in $A$.

This will certainly make sure that $T^{X}$ is Suslin whenever $X$ is stationary. Let us turn to the construction when $\beta$ is a limit ordinal from $\omega_{1} \backslash X$.

First, a new definition. Given any tree $T$ of height $\beta \leq \omega_{1}$, we say that $(\varphi, \delta)$ is a specializing pair on $T$ if

(i) $\varphi: T \rightarrow \mathbb{Q}$ is monotone (in particular, $T$ is special),

(ii) if $s<t \in T$ then $|\varphi(s)-\varphi(t)|<\delta(s)$, and

(iii) if $\alpha<\beta<\operatorname{ht}(T), s \in T_{\alpha}$ and $\Delta>0$ then there is some $t \in T_{\beta}$ above $s$ so that

$$
|\varphi(s)-\varphi(t)|<\Delta \text { and } \delta(t)<\Delta \text {. }
$$

Our first goal is the following: given a countable tree $T$ of limit height $\beta$ and a countable family $\Phi$ of specializing pairs for $T$, we show that there is a cofinal branch $b$ through $T$ so that any $(\varphi, \delta) \in \Phi$ can be extended to $t^{*}=\cup b$.

Lemma 3.6. Suppose that $T \subset \omega^{<\omega_{1}}$ is a countable tree of limit height $\beta$ and $\Phi$ is a countable family of specializing pairs for T. Fix some $s \in T,\left(\varphi^{*}, \delta^{*}\right) \in \Phi$ and $\Delta>0$. Then there is a cofinal, downward closed branch $b \subset T$ containing $s$ so that

$$
\sup _{s \leq t \in b}\left|\varphi^{*}(s)-\varphi^{*}(t)\right|<\Delta
$$

and for any $t^{\prime} \in b^{\downarrow}$ and $(\varphi, \delta) \in \Phi$,

$$
\sup _{t^{\prime} \leq t \in b}\left|\varphi\left(t^{\prime}\right)-\varphi(t)\right|<\delta\left(s^{\prime}\right) .
$$

Indeed, if the lemma holds and we set $t^{*}=\cup b$ and define $\varphi\left(t^{*}\right)=\sup _{t \in b} \varphi(t)$ for $(\varphi, \delta) \in \Phi$ then $(\varphi, \delta)$ still satisfies (i) and (ii) from the definition of a specializing pair on the extra node $t^{*}$. Moreover, if we set $\delta^{*}\left(t^{*}\right)=\Delta / 2$ then for this particular $s$ and $\Delta$, we made sure that condition (iii) for $\left(\varphi^{*}, \delta^{*}\right)$ is witnessed by $t^{*}$. By repeating the procedure of Lemma 3.6 for all the elements of $\Phi$ with all possible rational $\Delta>0$, we get the following.

Lemma 3.7. Suppose that $T \subset \omega^{<\omega_{1}}$ is a countable tree of limit height $\beta$ and $\Phi$ is a countable family of specializing pairs for $T$. Then $T$ has a pruned end-extension $T^{*}$ of height $\beta+1$ so that any $(\varphi, \delta) \in \Phi$ has an extension $(\bar{\varphi}, \bar{\delta})$ that is a specializing pair on $T^{*}$.

Proof of Lemma 3.6. Given $s<t \in T$ and $\mathbf{p} \in \Phi$, let $\Delta_{\mathbf{p}}(s, t)=\delta(s)-|\varphi(s)-\varphi(t)|$. This measures how much slack we have after jumping from $s$ to $t$. List all $\mathbf{p} \in \Phi$ and 
$t \in T$ as $\left(\mathbf{p}_{n}, t_{n}\right)$, each infinitely often. We define $s<s_{0}<s_{1}<s_{2}<\ldots$ so that $s_{n} \in T_{\alpha_{n}}$ for some fixed $\left(\alpha_{n}\right)_{n \in \omega}$ cofinal sequence in $\alpha$. First, we pick $s_{0}$ so that

$$
\varphi^{*}\left(s_{0}\right)-\varphi^{*}(s)<\Delta / 2
$$

and

$$
\delta^{*}\left(s_{0}\right)<\Delta / 2 .
$$

This can be done by (iii). Moreover, note that no matter how we pick $t$ above $s_{0}$, we will always have $\varphi^{*}(t)-\varphi^{*}\left(s_{0}\right)<\Delta / 2$ and so for any cofinal branch $b$ above $s_{0}$,

$$
\sup _{s \leq t \in b}\left|\varphi^{*}(t)-\varphi^{*}(s)\right|<\Delta
$$

by the triangle inequality.

Given $s_{n}$, we pick $s_{n+1}$ as follows: look at $\left(\mathbf{p}_{n}, t_{n}\right)$ and assume $t_{n}<s_{n}$ (if the latter fails, pick $s_{n+1}$ arbitrarily). Now, look at $\tilde{\Delta}=\Delta_{\mathbf{p}_{n}}\left(t_{n}, s_{n}\right)$ and pick $s_{n+1}$ so that

$$
\varphi_{n}\left(s_{n+1}\right)-\varphi_{n}\left(s_{n}\right)<\tilde{\Delta} / 2
$$

and

$$
\delta_{n}\left(s_{n+1}\right)<\tilde{\Delta} / 2
$$

where $\mathbf{p}_{n}=\left(\varphi_{n}, \delta_{n}\right)$. This is again possible by (iii). As before, for any $t$ above $s_{n+1}$, we will always have $\varphi_{n}(t)-\varphi_{n}\left(s_{n+1}\right)<\tilde{\Delta} / 2$ and so for any cofinal branch $b$ above $s_{n+1}$,

$$
\sup _{s_{n+1} \leq t \in b} \varphi_{n}(t)-\varphi_{n}\left(t_{n}\right)<\delta_{n}\left(t_{n}\right)
$$

by the triangle inequality and unwrapping the definition of $\tilde{\Delta}$.

The final branch $b$ is given by the downward closure of $\left(s_{n}\right)_{n<\omega}$. Since any $t^{\prime} \in b$ and specializing pair $\mathbf{p} \in \Phi$ was considered infinitely often during the construction, we clearly satisfied the requirements.

Finally, we can describe what happens in the construction of $T_{\beta}^{X}$ at limit steps $\beta \in \omega_{1} \backslash X$. Working in $N_{\beta+1}$, we consider the tree $T_{<\beta}^{X}$ and $\Phi_{\beta}=\left\{(\varphi, \delta) \in N_{\beta}:(\varphi, \delta)\right.$ is a specializing pair for $\left.T_{<\beta}^{X}\right\}$. Now, applying Lemma 3.7 we add a new level to $T_{<\beta}^{X}$ so that any specializing pair from $\Phi_{\beta}$ extends to $T_{\leq \beta}^{X}$.

This ends the construction of $T^{X}$ and we are left to prove the following.

Claim 3.8. If $X$ is non-stationary then $T^{X}$ is a special Aronszajn tree.

Proof. In fact, we prove that $T^{X}$ has a specializing pair. By our assumption on $X$, we can find a club $C \subset \omega_{1} \backslash X$ so that for any $\beta \in C, X \cap \beta, C \cap \beta \in N_{\beta}$. Let $\left\{\beta_{\alpha}: \alpha<\omega_{1}\right\}$ be the increasing enumeration of $C$ and we define an $\left(\varphi_{\alpha}, \delta_{\alpha}\right)$ for $\alpha<\omega_{1}$ so that

(1) $\left(\varphi_{\alpha}, \delta_{\alpha}\right) \in N_{\beta_{\alpha}+1}$ is a specializing pair on $T_{<\beta_{\alpha}}^{X}$ uniquely definable from $X \cap$ $\beta_{\alpha}, C \cap \beta_{\alpha}, \bar{N} \uparrow \beta_{\alpha}$,

(2) for $\alpha<\alpha^{\prime}<\omega_{1},\left(\varphi_{\alpha^{\prime}}, \delta_{\alpha^{\prime}}\right)$ extends $\left(\varphi_{\alpha}, \delta_{\alpha}\right)$.

As before, $N_{\beta_{\alpha^{\prime}}}$ has all the information to reconstruct the sequence $\left(\left(\varphi_{\alpha}, \delta_{\alpha}\right)\right)_{\alpha<\alpha^{\prime}}$ and so

$$
\left(\bigcup_{\alpha<\alpha^{\prime}} \varphi_{\alpha}, \bigcup_{\alpha<\alpha^{\prime}} \delta_{\alpha}\right) \in \Phi_{\beta_{\alpha^{\prime}}}
$$


Since $\beta_{\alpha^{\prime}} \notin X$, we made sure that this specializing pair has an extension to level $T_{\beta_{\alpha^{\prime}}}^{X}$ which gives $\left(\varphi_{\alpha^{\prime}}, \delta_{\alpha^{\prime}}\right)$.

Corollary 3.9. If $V=L$ then $\mathrm{sAT}$ is $\Sigma_{1}^{1}$-complete.

\section{Kurepa trees}

Our goal in this section is to show the following.

Theorem 4.1. $(V=L)$ The set $\mathrm{KT}$ of all Kurepa trees is $\Pi_{2}^{1}$-complete.

We prove the above result through a series of lemmas. First, we will build on the following representation of $\Pi_{2}^{1}$ sets.

Lemma 4.2. If $A$ is a $\Pi_{2}^{1}$ subset of $\omega_{1} \omega_{1}$ then for some $\Sigma_{1}$ formula $\phi$ and some parameter $P \in \omega_{1}{ }^{\omega_{1}}$, the following are equivalent

(1) $X \in A$,

(2) $\sup \left\{i<\omega_{2}: L_{\omega_{2}}[X] \models \phi(X, P, i)\right\}=\omega_{2}$.

Proof. Since $A$ is $\Pi_{2}^{1}$, we can find a Borel set $B$ so that $X$ is in $A$ if and only if

$$
\forall Y \exists Z(X, Y, Z) \in B .
$$

Let $f: \omega_{2} \rightarrow \omega_{1} \omega_{1}$ be a bijection which is $\Sigma_{1}$ over $L_{\omega_{2}}$ and choose a $\Sigma_{1}$ formula $\psi$ with parameter $P$ in $\omega_{1}{ }^{\omega_{1}}$ so that $(X, Y, Z) \in B$ if and only if $L_{\omega_{2}} \models \psi(X, Y, Z, P)$.

Then $X \in A$ if and only if

$$
L_{\omega_{2}} \models \forall j \exists k \psi(X, f(j), f(k), P) .
$$

Let $\phi(X, P, i)$ be the formula

$$
X, P \in L_{i} \wedge L_{i} \models \forall j \exists k \psi(X, f(j), f(k), P) .
$$

Then (4.1) (and so $X \in A$ as well) is equivalent to

as desired.

$$
\sup \left\{i<\omega_{2}: L_{\omega_{2}} \models \phi(X, P, i)\right\}=\omega_{2},
$$

Fix some $X \subset \omega_{1}^{\omega_{1}}$ with corresponding $\Sigma_{1}$ formula $\phi$ and parameter $P$. First, note that

$$
\left\{i<\omega_{2}: L_{\omega_{2}} \models \phi(X, P, i)\right\}=\left\{i<\omega_{2}: \exists \beta<\omega_{2}\left(L_{\beta} \models \phi(X, P, i)\right)\right\} .
$$

Our plan is to form an $\aleph_{1}$-tree $T=T_{X}$ consisting of triples $(\bar{\alpha}, \bar{i}, \bar{\beta})$ from $\omega_{1}$ which resemble a triple $\left(\omega_{1}, i, \beta\right)$ from $\omega_{2}$ with $L_{\beta} \models \phi(X, P, i)$. Distinct uncountable branches in the tree $T$ will correspond to distinct triples $\left(\omega_{1}, i, \beta\right)$. In turn, whether $T$ has $\omega_{2}$ many branches (i.e., if $T$ is Kurepa) will characterize whether $X \in A$. This will prove that $\mathrm{KT}$ is $\Pi_{2}^{1}$-complete.

We will say that a triple $(\alpha, i, \beta)$ from $\omega_{2}$ is good (with respect to $X, \phi$ and $P$ ) if

(1) $\alpha<i<\beta<\omega_{2}$,

(2) $L_{\beta} \models \alpha=\omega_{1}$,

(3) $\beta$ is the least limit ordinal so that
(a) $X \cap \alpha, P \cap \alpha \in L_{\beta}$
(b) $L_{\beta} \models|i|=\alpha$, 
(c) $L_{\beta}=\phi(X \cap \alpha, P \cap \alpha, i)$.

Note that $\alpha \leq \omega_{1}$; in case of equality, $X \cap \alpha=X$ and $P \cap \alpha=P$. If $\alpha<\omega_{1}$ then $\beta<\omega_{1}$ as well by (2).

The next claim should be clear from the minimality of $\beta$.

Claim 4.3. If $(\alpha, i, \beta)$ is good then the Skolem hull of $\alpha \cup\{\alpha, X \cap \alpha, P \cap \alpha, i\}$ in $L_{\beta}$ is all of $L_{\beta}$.

Next, we define an ordering on good triples: we write

$$
(\bar{\alpha}, \bar{i}, \bar{\beta}) \triangleleft(\alpha, i, \beta)
$$

if $\bar{\alpha}<\alpha$ and there is a (unique) elementary embedding

$$
\psi: L_{\bar{\beta}} \hookrightarrow L_{\beta}
$$

so that

(1) $\psi \uparrow \bar{\alpha}$ is the identity,

(2) $\psi(\bar{\alpha})=\alpha$,

(3) $\psi(X \cap \bar{\alpha})=X \cap \alpha, \psi(X \cap \bar{\alpha})=P \cap \alpha$ and

(4) $\psi(\bar{i})=i$.

Note that $L_{\bar{\beta}}=\psi^{-1}\left(L_{\beta}\right)$ is the transitive closure of $H=\operatorname{Hull}(\bar{\alpha} \cup\{\alpha, X \cap \alpha, P \cap \alpha\})$ in $L_{\beta}$. In turn, for a given good triple $(\alpha, i, \beta)$ and $\bar{\alpha}<\alpha$, there is $\bar{i}, \bar{\beta}$ so that $(\bar{\alpha}, \bar{i}, \bar{\beta})<(\alpha, i, \beta)$ iff for the above hull $H, H \cap \alpha=\bar{\alpha}$.

Let us summarize the basic properties of the relation $\triangleleft$.

Claim 4.4. (1) The relation $\triangleleft$ is transitive.

Moreover, for any good triple $(\alpha, i, \beta)$,

(2) for any $\bar{\alpha}<\alpha$, there is at most one choice of $\bar{i}, \bar{\beta}$ so that $(\bar{\alpha}, \bar{i}, \bar{\beta}) \triangleleft(\alpha, i, \beta)$;

(3) the set

is closed in $\alpha$.

$$
\{\bar{\alpha}<\alpha: \exists \bar{i}, \bar{\beta}(\bar{\alpha}, \bar{i}, \bar{\beta}) \triangleleft(\alpha, i, \beta)\}
$$

Claim 4.5. The relation $\triangleleft$ is a tree order on good triples.

Proof. Suppose that we are given good triples $\left(\alpha_{k}, i_{k}, \beta_{k}\right)$ for $k<3$ so that

$$
\left(\alpha_{0}, i_{0}, \beta_{0}\right),\left(\alpha_{1}, i_{1}, \beta_{1}\right) \triangleleft\left(\alpha_{2}, i_{2}, \beta_{2}\right) .
$$

If $\alpha_{0}=\alpha_{1}$ then $\left(\alpha_{0}, i_{0}, \beta_{0}\right)=\left(\alpha_{1}, i_{1}, \beta_{1}\right)$; indeed, this follows from the Claim 4.4 So we can assume $\alpha_{0}<\alpha_{1}$. Now, if $\psi_{j}: L_{\beta_{j}} \hookrightarrow L_{\beta_{2}}$ witnesses that $\left(\alpha_{j}, i_{j}, \beta_{j}\right) \triangleleft$ $\left(\alpha_{2}, i_{2}, \beta_{2}\right)$ for $j=0,1$ then $\psi=\psi_{1}^{-1} \circ \psi_{0}$ witnesses $\left(\alpha_{0}, i_{0}, \beta_{0}\right) \triangleleft\left(\alpha_{1}, i_{1}, \beta_{1}\right)$.

For some technical reasons, instead of taking the tree of good triples, we will look at functions associated to good triples and the tree formed by them. For each good triple $(\alpha, i, \beta)$, define a function $f_{(\alpha, i, \beta)}$ with domain $\alpha+1$ as follows:

$$
f_{(\alpha, i, \beta)}(\bar{\alpha})= \begin{cases}(\alpha, i, \beta) & \text { if } \bar{\alpha}=\alpha, \\ (\bar{\alpha}, \bar{i}, \bar{\beta}) & \text { if }(\bar{\alpha}, \bar{i}, \bar{\beta}) \triangleleft(\alpha, i, \beta) \text { for some } \bar{i}, \bar{\beta} \text {, and } \\ 0 & \text { otherwise. }\end{cases}
$$

This is well-defined by Claim 4.4. 
Claim 4.6. For any $(\bar{\alpha}, \bar{i}, \bar{\beta}) \triangleleft(\alpha, i, \beta), f_{(\alpha, i, \beta)}\left\lceil\bar{\alpha}+1=f_{(\bar{\alpha}, \bar{i}, \bar{\beta})}\right.$.

Proof. This follows immediately from the fact that $\triangleleft$ is a tree order.

We let $T_{X}$ be the set of function $f_{(\alpha, i, \beta)}\lceil\bar{\alpha}+1$ where $(\alpha, i, \beta)$ is a good triple (with respect to $X, \phi, P$ ) of countable ordinals and $\bar{\alpha} \leq \alpha$.

Claim 4.7. $T_{X}$ is a tree of height at most $\omega_{1}$ and countable levels.

Proof. We prove that every level of $T_{X}$ is countable by induction. Elements of $T_{X}$ at level $\bar{\alpha}$ are of the form $f=f_{(\alpha, i, \beta)}\lceil\bar{\alpha}+1$. These functions either satisfy (i) $f(\bar{\alpha})=0$ or (ii) $f(\bar{\alpha})=(\bar{\alpha}, \bar{i}, \bar{\beta})$. In case (i), $f$ must be constant 0 on an end-segment of $\bar{\alpha}$ and so $f$ is completely determined by the previous levels so by induction, there are only countably many choices for $f$. In case (ii), we note that $f=f_{(\bar{\alpha}, \bar{i}, \bar{\beta})}$ by Claim 4.6. Finally, note that there are only countably many possibilites for the value of $\bar{\beta}$ since for any large enough $\gamma<\omega_{1}, L_{\gamma} \models|\alpha| \leq \aleph_{0}$. This proves that level $\bar{\alpha}$ of $T_{X}$ must be countable.

The next lemma will conlcude the proof of the theorem.

Claim 4.8. $T_{X}$ has $\aleph_{2}$ uncountable branches if and only if the set

$$
\sup \left\{i<\omega_{2}: L_{\omega_{2}}[X] \models \phi(X, P, i)\right\}=\omega_{2} .
$$

Proof. First, assume that $L_{\omega_{2}} \models \phi(X, P, i)$ for some $i>\omega_{1}$. Pick the minimal $\beta$ so that $L_{\beta} \models \phi(X, P, i)$ and so $\left(\omega_{1}, i, \beta\right)$ is a good triple with respect to $X, \phi, P$.

Subclaim 4.8.1. $B=\left\{f_{\left(\omega_{1}, i, \beta\right)}\left\lceil\alpha+1: \alpha<\omega_{1}\right\}\right.$ is an uncountable branch in $T_{X}$.

Moreover, the branch $B$ uniquely determines the triple $\left(\omega_{1}, i, \beta\right)$ by the following claim.

Subclaim 4.8.2. $\left(L_{\beta}, X, P, i\right)$ is the direct limit of $\left(L_{\bar{\beta}}, X \cap \alpha, P \cap \alpha, \bar{i}\right)$ for $(\bar{\alpha}, \bar{i}, \bar{\beta}) \triangleleft$ $\left(\omega_{1}, i, \beta\right)$.

Thus distinct good triples $\left(\omega_{1}, i, \beta\right)$ correspond to different branches in $T_{X}$.

Conversely, suppose that we have a branch $B$ in $T_{X}$, which is not eventually 0 . Now, the direct limit of the $\left(L_{\bar{\beta}}, X \cap \bar{\alpha}, P \cap \bar{\alpha}, \bar{i}\right)$ for $(\bar{\alpha}, \bar{i}, \bar{\beta}) \in \operatorname{ran} \cup B$ yields some $\left(L_{\beta}, X, P, i\right)$ and a good triple $\left(\omega_{1}, i, \beta\right)$. Moreover, $B$ is the restriction of $f_{\left(\omega_{1}, i, \beta\right)}$. Distinct $\omega_{1}$-branches yield distinct triples $\left(\omega_{1}, i, \beta\right)$ and since $\beta$ is uniquely determined by $i$, we get $\omega_{2}$-many $i$ such that $\phi(X, P, i)$ holds in $L_{\omega_{2}}$.

\section{Open PROBLEMS AND FUTURE GOALS}

A positive answer to the following question would show that there are no ZFC reductions between stationarity and AT.

Question 5.1. Is it consistent with $\mathrm{CH}$ that all Aronszajn trees are special and there are no Canary trees?

Regarding Kurepa-trees, the following remains open.

Question 5.2. Can $\mathrm{KT}$ be $\Delta_{2}^{1}$ and nonempty? 
Question 5.3. What is the complexity of $\mathrm{KT}$ under $M A_{\aleph_{1}}$ (given such trees exist)?

The following would also be very interesting.

Question 5.4. Find a natural class of structures $\mathcal{X}$ in $\Sigma_{1}^{1} \backslash \Delta_{1}^{1}$ or $\Pi_{1}^{1} \backslash \Delta_{1}^{1}$ which is not complete for its complexity class.

The reason we ask for a natural class is that under $V=L$, one can build such artificial examples (and for inaccessible cardinals there are even natural examples) but we wonder if there are more combinatorial examples on $\omega_{1}$. Also, Harrington proved that consistently no such intermediate classes exist.

Yet another axiom to consider in more detail is $\operatorname{PFA}(S)$ for coherent Suslin-trees $S$ (see e.g., 222). Such models allow the existence of Suslin-trees while share many properties with models of the proper forcing axiom.

Question 5.5. How does PFA(S) affect the complexity of the classes AT, sAT and ST?

Once we force with the Suslin tree $S$ over a model of $P F A(S)$, the resulting extension has no Suslin trees any more and in fact, any two Aronszajn trees will be club-isomorphic 25]. In turn, the complexity of AT and sAT is $\Delta_{1}^{1}$ as mentioned in Figure 2 .

It would certainly be interesting to see to what extent our results generalise to higher cardinals above $\omega_{1}$. In particular, we mention a recent result of Krueger 13 on the club-isomorphism of higher Aronszajn trees that could substitute the AbrahamShelah model. The construction schemes developed by Brodsky, Lambie-Hanson and Rinot [4, 18, 15] for higher Suslin and Aronszajn trees also seems rather relevant. The theorem of Jensen on $\mathrm{CH}$ and all Aronszajn trees being special was recently generalized to higher cardinals in a breakthrough result by Asperó and Golshani [2]. Definability of $\mathrm{NS}_{\kappa}$ on successor cardinals was investigated by Friedman, Wu and Zdomskyy 9].

We believe that a similar analysis of other classes of structures on $\omega_{1}$ is well worth exploring. To name the most natural candidates, we would be interested in the following:

(1) Graphs and more generally colourings $c:\left[\omega_{1}\right]^{2} \rightarrow r$ with $r \leq \omega_{1}$. One might look at graphs with chromatic or colouring number $\omega_{1}$; or strong colourings that witness the failure of square bracket relations (i.e., $\omega_{1} \nrightarrow \nrightarrow\left[\omega_{1}\right]_{\omega_{1}}^{2}$ ). Hypergraphs and various set-systems are also natural candidates.

(2) Ladder systems on $\omega_{1}$. Natural classes are ladder systems with various guessing properties (i.e., o sequences or club guessing sequences); and ladder systems with or without the uniformization property.

(3) Linear orders on $\omega_{1}$. For Aronszajn and Suslin-lines, our analysis most likely yields the appropriate complexities but we did not address the important class of Countryman lines.

(4) Forcing notions. We can consider various classes of forcing posets on $\omega_{1}$ such as ccc, Knaster, $\sigma$-centered, $\sigma$-linked or proper partial orders.

Finally, it will be very natural to consider the classical equivalence relations on these classes and to find Borel definable reductions between them. To mention a few, we 
name the order isomorphism of trees and linear orders; the notion of club-isomorphism between trees; graph isomorphism; bi-embeddability of various structures; or forcing equivalence of posets.

\section{REFERENCES}

[1] Uri Abraham and Saharon Shelah. Isomorphism types of Aronszajn trees. Israel Journal of Mathematics, 50(1):75-113, 1985.

[2] David Asperó and Mohammad Golshani. The special Aronszajn tree property at $\aleph_{2}$ and GCH. arXiv preprint arXiv:1809.07638, 2018.

[3] James Baumgartner, Jerome Malitz, and William Reinhardt. Embedding trees in the rationals. Proceedings of the National Academy of Sciences, 67(4):1748-1753, 1970.

[4] Ari Meir Brodsky and Assaf Rinot. A microscopic approach to souslin-tree constructions, part i. Annals of Pure and Applied Logic, 168(11):1949-2007, 2017.

[5] Keith J Devlin and Havard Johnsbraten. The Souslin problem, volume 405. Springer, 2006.

[6] M. Dzamonja and J. Väänänen. A family of trees with no uncountable branches. Topology Proc., 28(1):113-132, 2004. Spring Topology and Dynamical Systems Conference.

[7] Ekaterina Fokina, Sy-David Friedman, Julia Knight, and Russell Miller. Classes of structures with universe a subset of $\omega_{1}$. Journal of Logic and Computation, 23(6):1249-1265, 2013.

[8] Sy-David Friedman, Tapani Hyttinen, and Vadim Kulikov. Generalized descriptive set theory and classification theory, volume 230. American Mathematical Society, 2014.

[9] Sy-David Friedman, Liuzhen Wu, and Lyubomyr Zdomskyy. $\delta_{1}$-definability of the non-stationary ideal at successor cardinals. Fundamenta Mathematicae, 229:231-254, 2015.

[10] Tapani Hyttinen and Mika Rautila. The canary tree revisited. The Journal of Symbolic Logic, 66(4):1677-1694, 2001.

[11] T Jech 3rd. Set theory, 3rd millennium ed, rev. and expanded ed, 2003.

[12] Alexander S Kechris and Alain Louveau. Descriptive set theory and the structure of sets of uniqueness, volume 128. Cambridge University Press, 1987.

[13] John Krueger. Club isomorphisms on higher Aronszajn trees. Annals of Pure and Applied Logic, 169(10):1044-1081, 2018.

[14] K. Kunen. Set theory an introduction to independence proofs. Elsevier, 2014.

[15] Chris Lambie-Hanson. Aronszajn trees, square principles, and stationary reflection. Mathematical Logic Quarterly, 63(3-4):265-281, 2017.

[16] Alan Mekler and Jouko Väänänen. Trees and $\Pi_{1}^{1}$-subsets of $\omega_{1} \omega_{1}$. J. Symbolic Logic, 58(3):10521070, 1993.

[17] Alan H Mekler and Saharon Shelah. The canary tree. Canadian mathematical bulletin, 36(2):209-215, 1993.

[18] Assaf Rinot. Higher Souslin trees and the gch, revisited. Advances in Mathematics, 311:510-531, 2017.

[19] Saharon Shelah. Proper and improper forcing, volume 5. Cambridge University Press, 2017.

[20] Saharon Shelah and Jouko Väänänen. Stationary sets and infinitary logic. The Journal of Symbolic Logic, 65(3):1311-1320, 2000.

[21] Jack Silver. The independence of kurepaâĂŹs conjecture and two-cardinal conjectures in model theory. In Axiomatic Set Theory, volume 13, pages 383-390. Amer. Math. Soc Providence, RI, 1971.

[22] Franklin D Tall. PFA(S)[S] for the masses. Topology and its Applications, 232:13-21, 2017.

[23] S. Todorcevic. Trees and linearly ordered sets. Handbook of set-theoretic topology, pages 235-293, 1984.

[24] Jouko Väänänen. Games and trees in infinitary logic: A survey. In Quantifiers: Logics, Models and Computation, pages 105-138. Springer, 1995.

[25] Teruyuki Yorioka. Club-isomorphisms of Aronszajn trees in the extension with a suslin tree. 2017. 
(Sy-D. Friedman) Universität Wien, Kurt Gödel Research Center for Mathematical Logic, Wien, Austria

E-mail address: sdf@logic.univie.ac.at

(D.T. Soukup) Universität Wien, Kurt Gödel Research Center for Mathematical Logic, Wien, Austria

E-mail address, Corresponding author: daniel.soukup@univie.ac.at

URL: http://www.logic.univie.ac.at/ soukupd73/ 